\title{
Zur klinischen Bedeutung von Selen: Ein systematischer Review
}

\author{
Reinhard Saller1', Christine Römer-Lüthi², Reto Brignoli33, Remy Meier ${ }^{4}$ \\ 1 UniversitätsSpital Zürich, Institut für Naturheilkunde, $\mathrm{CH}$-Zürich; 2 Berner Fachhochschule Gesundheit, $\mathrm{CH}$-Bern; \\ 3 Reto Brignoli, Facharzt für Pharmakologie, CH-Rüti; 4 Remy Meier, Medizinische Universitätsklinik, Abteilung für Gastroenterologie, CH-Liestal
}

$\mathrm{s}$ elen wurde im Jahr 1817 von Jacob Berzelius entdeckt und als neues Element nach der Mondgöttin Selene benannt. Lange Zeit galt Selen

\section{Selen - ein wichtiges Antioxidans}

als giftig und krebserregend, bis es 1957 als essentielles Spurenelement eingestuft wurde.

Selen ist in verschiedenen Nahrungsmitteln enthalten, zum Beispiel in Getreide, Geflügel, Schalentieren, Knoblauch, Fleisch usw. [1]. Die Resorption von Selen in den Organismus wird durch die gleichzeitige Einnahme von Ascorbinsäure (Vitamin C) gefördert. Gleichzeitig reduziert Vitamin C die Selenausscheidung, was zu einem grösseren Selen-Pool führt [2].

\section{Metabolismus}

Selen kann als Selenocystein in diverse Selenoproteine bzw. Selen-Enzyme eingebaut werden [3]. Selenocystein entspricht der Aminosäure Cystein, in der das Schwefel-Atom durch Selen ersetzt ist. Eine Übersicht über die Selen-Enzyme und ihre Funktionen gibt Tabelle 1.

Neben vielen anderen Funktionen ist die Beteiligung an der Bildung von aktivem Schilddrüsenhormon (5'-Dejodinase) sowie am Schutz der Endothelzelle vor Peroxynitrit (Selenoprotein P) zu nennen. Selen-Enzyme halten die Redoxbalance im Gleichgewicht; sie wirken Veränderungen im Stoffwechsel entgegen, die durch endogenen und exogenen oxidativen Stress verursacht werden [4]. Dadurch wirkt Selen als Antioxidans (Reoxidation des reduzier-
Hintergrund: Das essentielle Spurenelement Selen ist in verschiedenen Nahrungsmitteln enthalten, zum Beispiel in Tomaten, Geflügel, Schalentieren, Knoblauch, Fleisch etc. Selen-Enzyme wirken Veränderungen im Stoffwechsel entgegen, die durch endogenen und exogenen oxidativen Stress verursacht werden. Dadurch wirkt Selen als Antioxidans. Zielsetzung: Erstellung eines systematischen Review zur klinischen Bedeutung von Selen. Methoden: Systematische Analyse und Bewertung von Humanstudien (prospektive Doppelblindstudien, epidemiologische und retrospektive Studien, kurzfristige biochemische/hämatologische Studien ( Surrogate Markers»)) der letzten 10 Jahre aus den gängigen elektronischen Datenbanken sowie der Angaben von Standardwerken und publizierten Monographien. Ergebnisse und Schlussfolgerungen: Selen-Mangelerscheinungen können bei schweren chronischen Krankheiten oder infolge von zu wenig Selen in der Nahrung entstehen, sind aber insgesamt sehr selten. Ein subklinischer Selenmangel ist vermutlich in Ländern mit selenarmen Böden oder bei einseitiger, selenarmer Ernährung häufig. Der Nutzen von Selen (eventuell in Kombination mit Zink) bei der Vorbeugung und als Adjuvans bei der Behandlung von Infekten ist noch nicht bestätigt. Ermutigend sind die Ergebnisse von Studien betreffend Senkung des Krebsrisikos durch Selen. Wahrscheinlich gehen tiefe Selenspiegel mit einem erhöhten Risiko für Lungen-, Prostata- und Darmkrebs einher. Selensupplemente scheinen bei Personen mit vermehrtem oxidativen Stress sowie bei älteren Patienten mit erhöhtem Infektionsrisiko angebracht. Die Ernährungsgesellschaften von Deutschland, Österreich und der Schweiz (DACH-Empfehlungen) empfehlen 30-70 $\mu \mathrm{g} \mathrm{Se-}$ len pro Tag für Männer und Frauen. Die höchste verträgliche Dosis wird auf $400 \mu \mathrm{g}$ pro Tag geschätzt, ab Dosen von täglich $900 \mu \mathrm{g}$ kann Selen toxisch wirken.

Schlüsselwörter: Spurenelement, Selen, Selenmangel, Ernährung

\section{A Systematic Review of the Clinical Significance of Selenium}

Background: Selenium is an essential trace element and is contained in many foods, such as tomatoes, poultry, crustaceans, garlic and meat. Enzymes containing selenium counteract metabolic changes caused by endogenous and exogenous oxidative stress. In this way, selenium acts as an antioxidant. Objective: To conduct a systematic review of the clinical significance of selenium. Methods: Systematic analysis and evaluation of human studies including prospective double-blind studies, epidemiological and retrospective studies, short term biochemical and haematological studies with surrogate markers - performed in the last 10 years and found in major electronic data bases, coupled to information in standard works and published monographs. Results and Conclusion: The symptoms of selenium deficiency may arise in severe chronic disease or from lack of selenium in the food. However, they are generally very rare. Subclinical selenium deficiency is thought to be frequent in countries with selenium-deficient soils or with imbalanced low selenium nutrition. The benefit of selenium (possibly in combination with zinc) in the prevention of infections or as an adjuvant in their treatment has not yet been confirmed. Studies on reducing the risk of cancer with selenium have given encouraging results. Low selenium concentrations are probably correlated with increased risks of lung, prostate and intestinal cancer. Selenium supplements appear to be sensible for people under increased oxidative stress and for older patients at increased risk of infection. The nutritional societies of Germany, Austria and Switzerland (DACH recommendations) recommend 30-70 $\mu \mathrm{g}$ selenium per day for men and women. The maximal tolerated daily dose is estimated to be $400 \mu \mathrm{g}$. Daily doses of $900 \mu \mathrm{g}$ selenium or more may be toxic.

Key words: Trace element, selenium, selenium deficiency, nutrition 
Tab. 1. Selenoproteine und ihre Funktion (Nach BRIELMEIER \& SCHMIDT*)

\begin{tabular}{|c|c|c|}
\hline Selenoprotein & übliche Bezeichnungen & Hauptfunktion \\
\hline GPx1 & cytosolische Glutathion-Peroxidase (cGPx) & \multirow[t]{4}{*}{ Reduktion von $\mathrm{H}_{2} \mathrm{O}_{2}$ und Alkyl-Hydroperoxiden } \\
\hline GPx2 & gastrointestinale Glutathion-Peroxidase (gi-GPx) & \\
\hline GPx3 & Plasma-Glutathion-Peroxidase (pGPx) & \\
\hline GPx6 & GPx3-Homolog & \\
\hline GPx4 & $\begin{array}{l}\text { Phospholipid-Hydroperoxid-GSH-Peroxidase } \\
\text { (PHGPx, mPHGPx, snGPx) }\end{array}$ & $\begin{array}{l}\text { Reduktion von lipophilen } \\
\text { Hydroperoxiden }\end{array}$ \\
\hline TR1 & cytosolische Thioredoxin-Reduktase & \multirow[t]{3}{*}{ Reduktion von oxidierten Thiolgruppen } \\
\hline TR2 & mitochondriale Thioredoxin-Reduktase & \\
\hline TR3 & Testis-spezifische Thioredoxin-Glutathion-Reduktase (TGR) & \\
\hline DI1, DI2, DI3 & $\begin{array}{l}\text { Typ I 5'-Dejodinase (5'-DI), Typ II 5'-Dejodinase (5'-DII), } \\
\text { Typ III 5'-Dejodinase (5'-DIII) }\end{array}$ & Stoffwechsel von Schilddrüsenhormonen \\
\hline SPS2 & Selenophosphat-Synthetase (SeID) & Bereitstellung von Selenophosphat \\
\hline $\mathrm{P}$ & Selenoprotein $\mathrm{P}$ & u.a. Selentransport \\
\hline $\mathrm{N}$ & Selenoprotein N & assoziiert mit Muskeldystrophie \\
\hline $\mathrm{R}$ & Methionin-Sulfoxid-Reduktase B & Reduktion von Protein-Methionin-Sufoxiden \\
\hline $\begin{array}{l}15 \mathrm{kDa}, 18 \mathrm{kDa}, \\
\mathrm{H}, \mathrm{I}, \mathrm{K}, \mathrm{M}, \mathrm{O}, \mathrm{S}, \\
\mathrm{T}, \mathrm{V}, \mathrm{W}\end{array}$ & $\begin{array}{l}\text { 15kDa-Selenoprotein, 18kDa-Selenoprotein, } \\
\text { unbekannte Selenoproteine }\end{array}$ & unbekannt \\
\hline
\end{tabular}

ten Glutathions durch die GlutathionPeroxidase). Es steht in enger metabolischer Beziehung zum Vitamin E.

\section{Aufnahme und Ausscheidung}

Die empfohlene tägliche Selenaufnahme beträgt für Männer und Frauen $55 \mu \mathrm{g}$, für Schwangere $60 \mu \mathrm{g}$ und für stillende Frauen $70 \mu \mathrm{g}$. Die höchste verträgliche Dosis wird auf $400 \mu \mathrm{g}$ pro Tag geschätzt [5]. Bei schwangeren Frauen ist die Ausscheidungsrate von Selen erhöht [6]. Die Empfehlung der Ernährungsgesellschaften von Deutschland, Österreich und der Schweiz (DACH-Empfehlungen) liegt bei 30$70 \mu \mathrm{g}$ pro Tag für Männer und Frauen.

Die Bioverfügbarkeit von Selen hängt von der Quelle ab. Fisch und Selenat $\left(\mathrm{Na}_{2} \mathrm{SeO}_{4}\right)$ sollen eine bessere Bioverfügbarkeit aufweisen als Bierhefe [7]. Allerdings liefert Letztere das biologisch direkt verwertbare Selenmethionin, das länger im Organismus verweilt [8]. Beim Menschen bewirkt die tägliche Aufnahme von $200 \mu \mathrm{g}$ Selen - unabhängig von der Selenquelle - eine vergleichbare Zunahme der Glutathion-Peroxidase-Aktivität [9]. Normale Selenspiegel liegen bei
$1 \mu \mathrm{mol} / \mathrm{L}$ (Plasma 0.75-1.35 $\mu \mathrm{mol} / \mathrm{L}$, Blut 1.1-2.5 $\mu \mathrm{mol} / \mathrm{L})$ [10].

Selen wird hauptsächlich als das pharmakologisch aktive Methylselenol im Harn ausgeschieden. Bei hohen Dosen erfolgt die Ausscheidung auch als Dimethylselenit über die Atemwege, was der Atemluft einen Knoblauchgeruch verleiht [11,12].

\section{Selenmangel}

Selen-Mangelerscheinungen sind bisher bei schweren chronischen Krankheiten beschrieben worden, die auch den Magen-Darm-Trakt betreffen; zum Beispiel langdauernde parenterale Ernährung (etwa bei Morbus Crohn [13], „Short Bowel Syndrom“ nach operativer Entfernung eines grossen Teils des Darms [14,15], chronischer Pankreatitis [16], Phenylketonurie [17] oder bei zystischer Fibrose [18].

Gewisse Fälle von tödlichen Erkrankungen des Herzmuskels (Kardiomyopathie) werden Selenmangel infolge von zu wenig Selen in der Nahrung zugeschrieben [19]. Die Keshan-Krankheit (benannt nach der chinesischen Provinz Keshan) ist eine Kardiomyopathie, bei der es im Herzmuskel zu
Gewebenekrosen kommt; diese Erkrankung wird mit Selenmangel - zumindest als prädisponierendem Faktor und viralen Infekten in Verbindung gebracht $[20,21,22]$. Man vermutet, dass die Vermehrung von Coxsackie-Viren, welche die Keshan-Krankheit auslösen können, durch Selen gehemmt wird [23].

Selenmangel dürfte auch bei der Kashin-Beck-Erkrankung eine Rolle spielen, die in Nordostasien vorkommt. Bei dieser Krankheit treten generalisierte Gelenksentzündungen und Wachstumsstörungen auf. Primär muss bei dieser Erkrankung aber der Jodmangel behoben werden [24].

Ein subklinischer Selenmangel ist vermutlich auch in verschiedenen anderen Ländern mit selenarmen Böden (Schweiz, Deutschland, Finnland) oder einseitiger, selenarmer Ernährung häufig. Verschiedene europäische Staaten bekämpfen diesen Mangel mit gezielten Massnahmen, Finnland zum Beispiel mit dem Zusatz von Selen zu Düngemitteln. In der Schweiz ist die Bevölkerung im Allgemeinen gut mit Selen versorgt [25]. Für eine ausreichende Versorgung von Mensch und Tier mit dem essentiellen Spurenele- 
ment Selen (Se) sind die natürlichen Umweltbedingungen in der Schweiz ungünstig. Eingehende Untersuchungen haben aber gezeigt, dass die Bevölkerung trotzdem ausreichend mit Selen versorgt ist. In den letzten 15 Jahren hat sich der Selenstatus der schweizerischen Bevölkerung nicht wesentlich verändert, da der tiefere Beitrag aus pflanzlichen Lebensmitteln zufälligerweise durch den höheren Beitrag aus tierischen Lebensmitteln kompensiert wurde [26].

\section{Selenvergiftung}

Selenvergiftungen (Selenosen) sind seit langem bei Tieren bekannt und treten in einigen Regionen der Erde auch bei Menschen auf. So gibt es zum Beispiel in China endemische Selenosen mit Selen-Blutspiegeln von über $100 \mu \mathrm{g} / \mathrm{dL}$. Die häufigsten Symptome einer Selenose sind Haarausfall und Verlust der Nägel. Hautläsionen und Entzündungen der Nerven lassen sich weniger sicher mit einer Selenvergiftung in Verbindung bringen.

Die Einnahme von über $900 \mu \mathrm{g}$ Selen pro Tag kann zu einer Selenvergiftung führen. Aus diesem Grund kann es sinnvoll sein, vor dem Verschreiben therapeutischer Dosen Selen den Selenspiegel im Blut zu überprüfen oder den Selengehalt von Haaren oder Nägeln zu messen (mittlere Werte von $0.86(0.76-4.3) \mu \mathrm{g} / \mathrm{g})$.

\section{Selen als Nahrungsergänzung oder als Therapeutikum}

In verschiedenen Arbeiten wurden die Wirkungen von Selen als Nahrungsergänzung (Supplementum) oder zur Behandlung von Krankheiten (Therapeutikum) untersucht. Für die Annahme, dass Selen einen vorbeugenden und/oder therapeutischen Effekt ausübt, sprechen verschiedene epidemiologische Studien. Die Ergebnisse dieser Studien werden als „vorläufig“ betrachtet. Sie können den routinemässigen Einsatz von Selen-Supplementen (noch) nicht rechtfertigen, besonders weil Selen in hohen Dosen toxisch wirken kann [27].

\section{Selen als Monotherapie}

Chronische entzündliche Prozesse können mit einer Selen-Monotherapie sehr wahrscheinlich günstig beeinflusst werden. Eine schützende Wirkung vor gewissen Krebsarten scheint ebenfalls wahrscheinlich. Die bisher publizierten Daten müssen aber durch grössere Studien noch erhärtet werden.

\section{Entzündliche und infektiöse Erkrankungen}

Tiefe Selenspiegel lassen sich nachweisen bei chronisch entzündlichen und infektiösen Erkrankungen, bei Hämodialyse von Intensivpatienten [28], bei Organversagen [29] und bei HIVInfektion respektive Aids-Erkrankung. Stark erniedrigte Selenspiegel sind mit einer erhöhten Erkrankungshäufigkeit und Sterblichkeit vergesellschaftet, eine kausale Verknüpfung wurde allerdings nicht nachgewiesen [30].

Sepsis: Die Gabe von Selen scheint die Häufigkeit von Komplikationen und Organversagen bei Sepsis und anderen systemischen Entzündungen zu reduzieren; schlüssige prospektive Studien fehlen allerdings noch [31,32].

Autoimmune Thyreoiditis (Schilddrüsenentzündung): In vergleichenden prospektiven Studien konnten bei autoimmuner Thyreoiditis mit Selen positive Resultate erreicht werden, das heisst eine Abnahme der ThyroidPeroxidase-Antikörper [33,34].

Asthma: Möglicherweise verbessert Selen auch die Befindlichkeit von Asthma-Patienten - allerdings nicht deren Atemfunktion [35,36,37].

Rheumatoide Arthritis: In einer Studie erhielten Patienten mit rheumatoider Arthritis täglich $200 \mu$ g Selen plus Fettsäuren aus Fischöl. Es zeigte sich eine signifikante Reduktion verschiedener Entzündungsparameter wie C-reaktives Protein, Prostaglandin E2 und Alpha-2-Globulinen sowie eine Verbesserung der klassischen klinischen Kriterien von rheumatoider Arthritis [38]. Wurde Selen als Monotherapie verabreicht, konnten die positiven Resultate nicht bestätigt werden [39].

Beeinflussung der Immunabwehr: Bei Probanden mit Selenspiegeln im unteren Normbereich (unter 1.2 umol/ L) konnte nach einer Impfung gegen Kinderlähmung eine Verstärkung der Immunreaktion (Interferon-gamma, THelferzellen etc.) nachgewiesen werden, wenn sie täglich $100 \mu \mathrm{g}$ Selen einnahmen [40]. Bei 60 Krebspatienten, die nach einer Operation unter einem sekundären Lymphödem litten, konnte die Häufigkeit von Hautinfekten (Erysipel) signifikant reduziert werden. In der Kontrollgruppe traten in drei Monaten bei 50 Prozent der Patienten Infekte auf, in der mit Selen behandelten Gruppe war kein einziger Patient von Infekten betroffen [41].

HIV-Infektion/Aids: In einer Studie in den USA nahmen HIV-Patienten zusätzlich zur üblichen Therapie pro Tag $200 \mu \mathrm{g}$ Selen ein. Im Vergleich zur Kontrollgruppe (Einnahme von Placebo) nahm die Hospitalisationsrate in der Verumgruppe deutlich ab und die Patienten fühlten sich auch psychisch besser $[42,43]$. In einer anderen Studie mit 262 Patienten beobachtete man nach neun Monaten Behandlung mit Selen eine Stabilisierung der Viruslast sowie eine signifikante Verbesserung der Anzahl CD4-Zellen [44]. Bei HIVinfizierten Frauen in Tansania stellte man fest, dass zwischen tiefen Selenspiegeln und erhöhter Sterblichkeit der Frauen und ihrer Kinder ein deutlicher Zusammenhang bestand $[45,46]$.

Pankreatitis: Eine Studie berichtet über den erfolgreichen Einsatz von Natriumselenit bei der akuten Pankreatitis. Bei initialer Gabe von 1000 $\mu \mathrm{g}$ Selen, gefolgt von einer täglichen Gabe von $500 \mu \mathrm{g}$ Selen als Erhaltungsdosis, sank die Sterblichkeit der Patienten auf weniger als zehn Prozent der Betroffenen [47]. In einer anderen Studie wurde versucht, mit der Gabe von Selen einer akuten Pankreatitis vorzubeugen, die manchmal als Folge einer Spiegelung der Gallen- und Pankreasgänge auftreten kann - jedoch ohne Erfolg [48].

Herz-Kreislauf-Krankheiten: In einer Studie wurden 60 Patienten, die einen Herzinfarkt erlitten hatten, während eines Jahrs mit täglich $100 \mu$ g Selen 
Tab. 2. Zusammenfassung der Studien bezüglich Selen und Lungenkrebs [aus Tabellen 1 und 4 in Cancer Epidemiol Biomarkers Prev 2004;13(5)]

\begin{tabular}{|c|c|c|c|c|c|c|c|c|}
\hline Autor & $\begin{array}{l}\text { Studien- } \\
\text { design }\end{array}$ & $\begin{array}{l}\text { Selen- } \\
\text { bestimmung }\end{array}$ & $\begin{array}{c}\text { Ge- } \\
\text { schlecht }\end{array}$ & $\begin{array}{l}\text { Studien- } \\
\text { ort }\end{array}$ & $\begin{array}{l}\text { Follow-up, } \\
\text { Jahre }\end{array}$ & $\begin{array}{l}\text { Anzahl } \\
\text { Fälle (n) }\end{array}$ & $\begin{array}{c}\text { RR } \\
(95 \% \mathrm{Cl})\end{array}$ & $\begin{array}{l}\text { P für } \\
\text { Konzentration } \\
\text { Wirkung }\end{array}$ \\
\hline Comstock et al. 1997 & NCC & Serum & Beide & $\begin{array}{c}\text { USA, } \\
\text { Maryland }\end{array}$ & $15-18$ & 258 & $0.65(0.41-1.02)$ & 0.08 \\
\hline Goodman et al. 2001 & $\mathrm{NCC}$ & Serum & Beide & USA & $5-14$ & 356 & $1.20(0.77-1.88)$ & 0.49 \\
\hline Kabuto et al. 1994 & $\mathrm{NCC}$ & Serum & Beide & Japan & $11-13$ & 77 & $0.56(0.20-1.43)$ & NA \\
\hline Knekt et al. 1990 & NCC & Serum & Männer & Finnland & 8-12 & 153 & $0.66(0.37-1.19)$ & 0.001 \\
\hline Knekt et al. 1998 & $\mathrm{NCC}$ & Serum & Beide & Finnland & $16-19$ & 77 & $0.41(0.17-0.94)$ & 0.46 \\
\hline Ratnasinghe et al. 2000 & NCC & Serum & Männer & China & $4-5$ & 108 & $1.20(0.60-2.40)$ & 0.52 \\
\hline Reid et al. 2002 & $\begin{array}{l}\text { Randomisierte } \\
\text { klinische Studie }\end{array}$ & Plasma & Beide & $\begin{array}{l}\text { Osten } \\
\text { der USA }\end{array}$ & $5-13$ & 60 & $0.74(0.44-1.24)$ & NA \\
\hline Garland et al. 1995 & NCC & Zehennagel & Frauen & USA & 3.5 & 47 & $4.33(0.54-34.60)$ & 0.17 \\
\hline Hartman et al. $2002 *$ & NCC & Zehennagel & Männer & Finnland & $5-8$ & $\begin{array}{c}\text { Keine } \\
\text { Angaben }\end{array}$ & $0.20(0.09-0.44)$ & NA \\
\hline Hartman et al. $2002 *$ & NCC & Zehennagel & Männer & Finnland & $5-8$ & $\begin{array}{c}\text { Keine } \\
\text { Angaben }\end{array}$ & $0.61(0.27-1.41)$ & NA \\
\hline $\begin{array}{l}\text { van den Brandt } \\
\text { al. } 1993\end{array}$ & $\begin{array}{l}\text { Kohorten- } \\
\text { studie }\end{array}$ & Zehennagel & Beide & Holland & 3.3 & 317 & $0.50(0.30-0.81)$ & $0.006 \mathrm{et}$ \\
\hline Hu et al. 1997 & $\begin{array}{c}\text { Fall-Kontroll- } \\
\text { Studie }\end{array}$ & $\mathrm{FFO}$ & Beide & China & - & 227 & $1.30(0.70-2.20)$ & 0.48 \\
\hline Kromhout 1987 & Kohortenstudie & FFQ & Männer & Holland & 25 & 63 & $0.98(0.41-2.36)$ & $>0.01$ \\
\hline Zhou et al. 1999 & Fall-Kontroll-Studie & FFO & Frauen & China & - & 290 & $0.76(0.47-1.15)$ & 0.11 \\
\hline \multicolumn{4}{|l|}{ Alle Studien kombiniert } & & & 2033 & $0.74(0.63-0.94)$ & \\
\hline \multirow[t]{5}{*}{$\begin{array}{l}\text { Studien kombiniert } \\
\text { nach }\end{array}$} & \multirow{3}{*}{$\begin{array}{l}\text { Selen- } \\
\text { Bestimmung }\end{array}$} & $\begin{array}{l}\text { Ernährungs- } \\
\text { anamnese }\end{array}$ & & 5 Studien & & 701 & $1(0.77-1.30)$ & \\
\hline & & Serum & & 6 Studien & & 1029 & $0.8(0.63-1.03)$ & \\
\hline & & Zehennagel & & 4 Studien & & 364 & $0.46(0.32-0.66)$ & \\
\hline & \multirow{2}{*}{$\begin{array}{l}\text { Selenspiegel in } \\
\text { der allgemeinen } \\
\text { Bevölkerung }\end{array}$} & Hoch & & 4 Studien & & 754 & $0.86(0.65-1.15)$ & \\
\hline & & Tief & & 6 Studien & & 855 & $0.72(0.56-0.93)$ & \\
\hline \multicolumn{9}{|c|}{$\begin{array}{l}\text { NCC: } \\
\text { FFQ: Fragebod case control } \\
\text { RR: relatives Risiko } \\
\text { * Früh randomisierte Patienten vs. Patienten, die nach } 5 \text { Jahren randomis }\end{array}$} \\
\hline
\end{tabular}

und 100 mg Coenzym-Q oder Placebo behandelt. Nach Ablauf dieses Jahres (Follow-up-Periode) war die Sterblichkeit in der Verumgruppe signifikant tiefer als in der Placebogruppe [49]. Andere Studien konnten diese Resultate jedoch nicht bestätigen.

In der wichtigen Health Professionals Follow-up Study untersuchte man, ob bei Personen mit einer geringen Selenversorgung gehäuft Herzkrankheiten auftreten. Dazu wurde die Selenkonzentration in den Zehennägeln bestimmt. Es ergab sich ein Zusammenhang zwischen tiefen Selenspie- geln und nicht-tödlichen Herzinfarkten, ein Zusammenhang zwischen tiefen Selenspiegeln und tödlichen Herzinfarkten liess sich aber nicht feststellen [50].

Die US-amerikanische Agency for Healthcare Research and Quality stufte Selen in ihrer im Mai 2006 erschienenen Gesamtbeurteilung von Vitaminen und Mineralstoffen als „unwirksam“ bezüglich kardiovaskulärer Erkrankungen ein [51,52].

\section{Vorbeugung von Krebs}

In der bereits erwähnten Gesamtbeur- teilung von Vitaminen und Mineralstoffen der Agency for Healthcare Research and Quality wurde die Evidenz, dass Selen eine präventive Wirkung gegenüber Karzinomen habe, als „moderat“ eingestuft [51]. In einer Kohortenstudie, die in Frankreich mit 1'389 älteren Teilnehmern durchgeführt wurde, ergab sich bei den Teilnehmern mit den anfänglich tieferen Selenspiegeln eine signifikant erhöhte Neun-Jahres-Mortalität (relatives Risiko 1.54) [53]; dieser Unterschied konnte spezifisch einer höheren Krebsmortalität zugeschrieben werden (RR 1.79). 
In der Nutritional Prevention of Cancer-Study bestand das ursprüngliche Ziel darin, bei Patienten mit einem malignen Melanom durch die Gabe von täglich $200 \mu \mathrm{g}$ Selen die Häufigkeit von anderen Hautkrebstypen zu reduzieren [54]. Dieses Ziel wurde zwar verfehlt, dafür ergab sich als Nebenbefund, dass die Häufigkeit von anderen Krebsarten eindeutig sank (relatives Risiko 0.55). Besonders deutlich war die Risikoreduktion bei Prostatakrebs $(\mathrm{RR}=0.37)$, beim kolorektalen Karzinom (RR $=0.42)$ und beim Lungenkarzinom $(\mathrm{RR}=0.54)$ [55].

Ein Mangel an selenhaltiger Glutathion-Peroxidase wurde auch bei der Entstehung von verschiedenen Karzinomen impliziert (Kopf-Hals-, Lungenund Brustkrebs) und dürfte auch bei einem grossen Teil der Kolonkarzinome von Bedeutung sein [56].

Lungenkrebs: Es liegen keine doppelblinden, prospektiven Interventionsstudien vor. Eine ausführliche MetaAnalyse der vorliegenden Beobachtungsstudien von ZHuo et al. zeigte insgesamt ein relatives Lungenkrebsrisiko von 0.74 bei den Probanden, die viel Selen einnahmen resp. hohe Selenkonzentrationen im Blut oder in den Zehennägeln aufwiesen [57]. Die Resultate sind allerdings widersprüchlich, wie in Tabelle 2 aufgeführt. Die Analyse weist auf verschiedene Faktoren hin, die das Resultat verfälschen könnten. Selenkonzentrationen, die in Nagelmaterial gemessen werden, scheinen geringeren Schwankungen zu unterliegen als Blut- oder Plasmaspiegel; daher haben Selenwerte, die in Nägeln gemessen werden, grösseren prädiktiven Wert. Die Wirkung von Selen-Supplementen zeigte sich deutlicher in Gegenden mit geringer Selenzufuhr (selenarme Böden).

Prostatakrebs: Es liegen keine doppelblinden, prospektiven Interventionsstudien vor; eine solche Studie läuft aber gegenwärtig [58]. Ob Selen vor Prostatakrebs schützt, wurde schon lange kontrovers diskutiert. Die relativen Chancen (Odds Ratio = Chancenverhältnis: Das Verhältnis des Odds für ein Therapieversagen/-erfolg unter der experimentellen Behandlung ge-

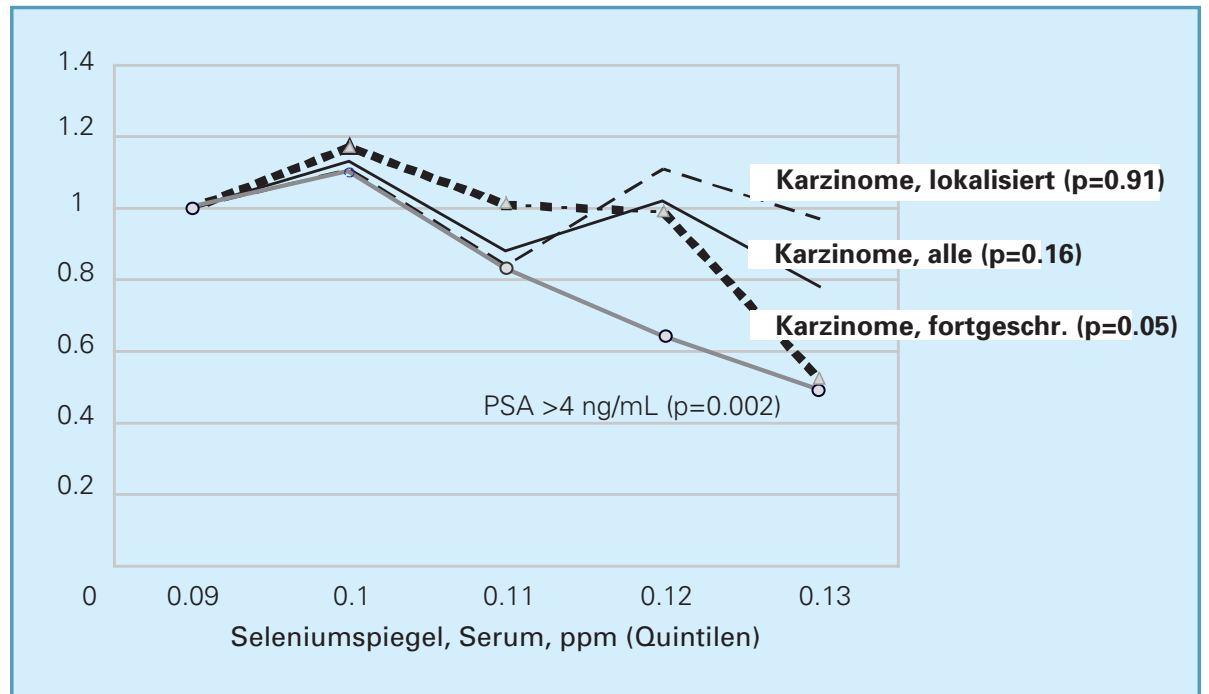

Abb. 1. Relative Chancen (OR) für ein Prostatakarzinom als Funktion der Selenspiegel (Physicians' Health Study), Selen als Kombinationsbehandlung.

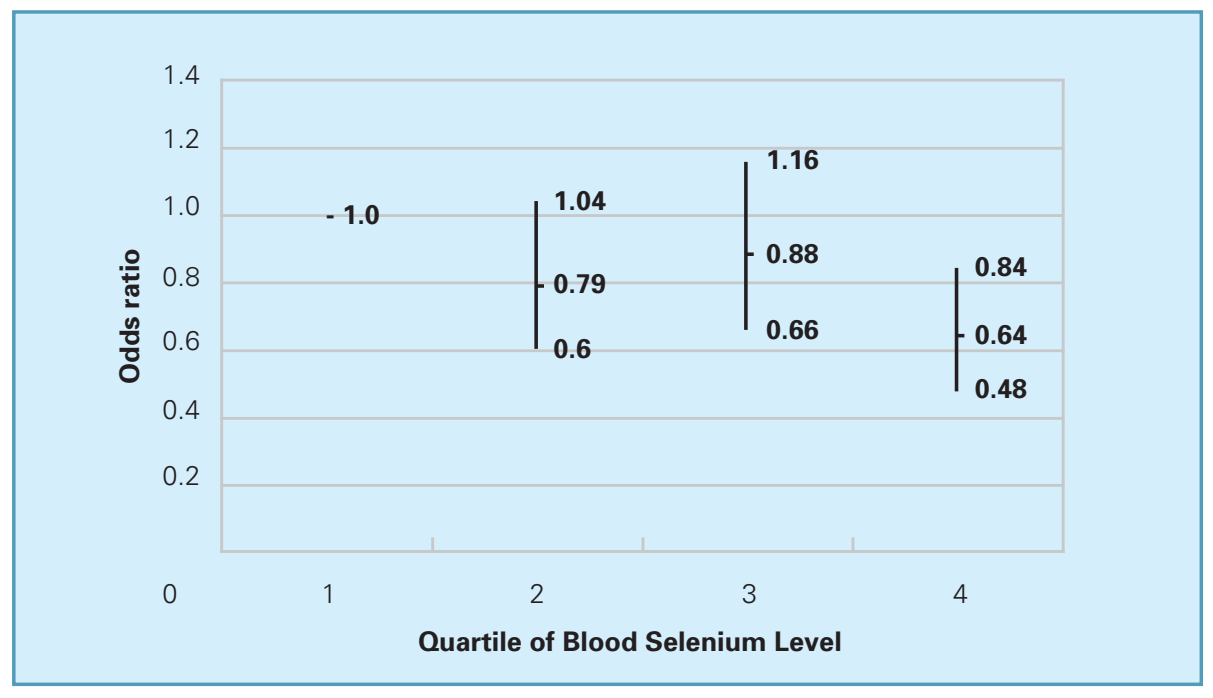

Abb. 2. Relative Chancen (OR) für ein Kolonkarzinom nach plasmatischen Selenspiegeln bei Aufnahme in die prospektiven Studien (JACOBS et al. [81]).

teilt durch das Odds für ein Therapieversagen/-erfolg unter der Standardbehandlung wird als Odds Ratio (OR) bezeichnet: $\mathrm{OR}=(\mathrm{a} / \mathrm{b}) /(\mathrm{c} / \mathrm{d})=(\mathrm{ad}) /(\mathrm{bc})$, wobei a bis d den vier Zellhäufigkeiten in einer Vierfeldertafel entsprechen. Bei einem Risiko < 0.10 scheint es gerechtfertigt, die Begriffe Odds und Risiko synonym zu verwenden.

In der Physicians' Health Study [59] wurde die Abhängigkeit der Erkrankung an einem Prostatkarzinom vom Selenspiegel untersucht. Die Chancenverhältnisse, an einem fortgeschrittenen Prostatakarzinom zu leiden, waren bei den Probanden mit tiefen Selenspiegeln erhöht (Abbildung 1).
Auch in der Netherlands Cohort Study, die 58'279 Männer einschloss, zeigte sich eine Abnahme der Prostatakrebsfälle parallel zur zunehmenden Selenkonzentration in den Zehennägeln (relatives Risiko 0.69) [60,61]. Die Schutzwirkung von Selen dürfte zumindest teilweise mit der Aktivität der prostatischen Glutathion-S-Transferase in Zusammenhang stehen, einem Enzym, das oxidative Schäden reduziert [62].

Tumoren im Magen-Darm-Trakt: Es liegen keine doppelblinden, prospektiven Interventionsstudien vor. Antioxidantien, wie Betacarotin oder die Vitamine $\mathrm{A}, \mathrm{C}$ und $\mathrm{E}$, schützen laut einer 
grossen Meta-Analyse nicht vor Krebs im Magen-Darm-Trakt; auch für Selen konnte eine Schutzwirkung nicht mit Sicherheit nachgewiesen werden [63]. Die Analyse von drei randomisierten prospektiven Studien mit 1'763 Adenom-Patienten zeigte, dass das Risiko für ein Kolonkarzinom in der Gruppe mit den anfänglich höchsten Selenspiegeln im Blut signifikant reduziert war (Abbildung 2) [64]. Chinesische Studien beschreiben, dass bei Personen mit höheren Selenspiegeln Leberkrebs sowie Magen- oder Speiseröhrenkrebs seltener auftreten [65, 66]. Die Studien weisen jedoch bedeutende methodologische Mängel auf [67].

Selen als Kombinationsbehandlung In den letzten Jahren wurden die Resultate verschiedener Studien veröffentlicht, welche die antioxidative Wirkung von Selen beim Menschen aufzeigen. Allerdings wurde in diesen Studien mit Kombinationspräparaten gearbeitet. Welche Komponente(n) dieser Präparate für die Wirkung verantwortlich ist, lässt sich deshalb nicht sicher eruieren.

In drei Studien, die mit wenigen Probanden durchgeführt wurden, verglich man die Kombination von Betacarotin, Vitamin C, Vitamin E und Selen bzw. Betacarotin, Vitamin C, Vitamin E und Coenzym Q10 mit Placebo. Es konnte eine Reduktion der Lipidperoxide nachgewiesen werden, in zwei Studien bei männlichen Patienten, in einer Studie bei Rauchern [68,69,70]. Diese Ergebnisse dürften auf einen klinischen Nutzen der entsprechenden Kombinationspräparate hinweisen.

In einer placebokontrollierten Studie mit 34 älteren Patienten, die während sechs Monaten mit täglich $100 \mu \mathrm{g}$ Selen und $20 \mathrm{mg}$ Zink behandelt wurden, beobachtete man einen Anstieg der Lycopen- und Glutathion-Peroxidase. Diese Enzyme dienen als Marker für eine antioxidative Wirkung [71].

In einer kleineren, prospektiven Studie erhielten Trauma-Patienten N-Acetylcystein, Selen und die Vitamine C und E. Die Häufigkeit von Komplikationen und multiplem Organversagen konnte eindeutig reduziert werden [72].

\section{Vorbeugung von Infektionen bei Risikopatienten}

In einer Schweizer Studie wurden schwer verbrannte Patienten mit einer Kombination von täglich $100 \mu \mathrm{g}$ Selen, $20 \mathrm{mg}$ Zink und $40.4 \mathrm{mmol}$ Kupfer behandelt; Ziel war die Vorbeugung von Infektionen. Tatsächlich traten bei den behandelten Patienten deutlich weniger Atemwegsinfektionen auf als bei den Patienten, die Placebo plus die Standardtherapie erhielten [73]. Die Autoren berichteten auch über eine etwas raschere Normalisierung der Schilddrüsenfunktion [74]. In einer anderen Studie senkte eine Tagesdosis von $100 \mu \mathrm{g}$ Selen und $20 \mathrm{mg}$ Zink die Infektionsrate bei älteren Patienten signifikant [75].

In einer doppelblinden Cross-OverStudie aus dem Jahr 1999 wurden ebenfalls verschiedene Kombinationspräparate geprüft [76]. Die ca. 360 älteren Studienteilnehmer erhielten während zwei Jahren eine der folgenden Kombinationen:

1. Selen und Zink

2. Betacarotin, Vitamin C und Vitamin E

3. Die Kombination von 1. und 2.

4. Placebo

Endpunkte der Studie waren die delayed-type hypersensitivity skin response (DTH), die humorale Antwort auf eine Grippeimpfung sowie die Morbidität und Mortalität infolge von Infektionskrankheiten. Keine der Therapien beeinflusste die DTH der Haut oder die Gesamtsterblichkeit. Bei den Patienten, die Selen und Zink erhielten (mit oder ohne zusätzliche Vitamine) war aber die humorale Reaktion auf die Impfung besser und es traten weniger Infektionen auf. Diese Resultate bestätigen die Ergebnisse einer früheren Studie, in welcher die Patienten ein Jahr lang mit Zink und Selen oder mit den Vitaminen C und E sowie Betacarotin behandelt worden waren [78].

\section{Verträglichkeit von Krebstherapien}

In einer prospektiven Studie mit 48 Krebspatienten versuchte man durch die Gabe von Selen, Vitamin C und Vitamin E die Nebenwirkungen der Chemotherapie mit Cisplatin zu reduzieren (ototoxische und nephrotoxische Wirkung); die Ergebnisse waren je- doch nicht eindeutig [78]. Eine andere Studie, bei der geprüft wurde, ob die Gabe von Selen die Verträglichkeit von Irinotecam verbessern könnte, lieferte keine positiven Resultate [79]. Diarrhö als Folge einer Radiotherapie soll dank einer Selentherapie reduziert werden; genaue Zahlen fehlen noch [80].

\section{Schlussfolgerungen}

\section{Nutzen von Selen}

- Unseres Erachtens belegen die vorhandenen Studien den Nutzen von Selen (eventuell in Kombination mit Zink) bei der Vorbeugung und als Adjuvans bei der Behandlung von Infekten noch nicht, da schlüssige prospektive Studien fehlen.

- Ermutigend - wenn auch noch bestätigungsbedürftig - sind bisher die Ergebnisse von Studien betreffend Senkung des Krebsrisikos durch Selen. Wahrscheinlich gehen tiefe Selenspiegel mit einem erhöhten Risiko für Lungen-, Prostataund Darmkrebs einher. Gross angelegte Studien zur Überprüfung dieser Daten (auch in Kombination mit Chemotherapie) sind im Gange.

- Ein Selensupplement wird oft bei Personen mit vermehrtem oxidativem Stress sowie bei älteren Patienten mit erhöhtem Infektionsrisiko empfohlen. Die diesbezügliche Datenlage ist jedoch noch kontrovers.

\section{Toxizität von Selen (Risiko-Nutzen-Analyse)}

- Im Dosierungsbereich von 50-200 $\mu \mathrm{g}$ pro Tag scheint Selen unbedenklich zu sein.

- Ob bei Risikopopulationen (ältere Patienten, Raucher etc.) der potentielle Nutzen eines Selensupplements grösser ist als das mögliche Risiko, kann noch nicht schlüssig beantwortet werden.

\section{Gebrauchsempfehlung}

- Empfohlen werden 50-200 $\mu$ g Selen pro Tag (durchschnittlich $100 \mu \mathrm{g}$ pro Tag; DACH-Empfehlung 30-70 $\mu \mathrm{g}$ pro Tag).

- Höhere Dosen von Selen sollten speziellen experimentellen Indikatio- 
nen vorbehalten bleiben und nur in einer kontrollierten Umgebung (Spital) verabreicht werden.

- Die Dauer der Behandlung sollte individuell angepasst werden. Daten über die Langzeittherapie gibt es (noch) nicht.

Die Autoren danken Herrn Prof. Dr. med. Paolo Suter, UniversitätsSpital Zürich, Abteilung für Hypertonie, für die vielen wertvollen Anregungen beim Verfassen des Manuskriptes.

This paper is an updated summary of an expert-workshop 2007 sponsored by BIOMED, $\mathrm{CH}$-Dübendorf.

\section{Literatur}

1 Mark H. Beers, Robert S. Porter, Thomas V. Jones, Justin L Kaplan, Michael Berkwits (Editors) The Merck Manual of Diagnosis and Therapy.: Section Nutritional Disorders; Subject Mineral Deficiency and Toxicity Topics · Selenium November 2005.

2 Martin RF Young VR Blumberg J Janghorbani $M$ Ascorbic acid-selenite interactions in humans studied with an oraldose of 74SeO3(2-). Am J Clin Nutr (1989 May); 49(5): 862-869

3 Dolph L. Hatfield1 and Vadim N. Gladyshev. How Selenium Has Altered Our Understanding of the Genetic Code. MOLECULAR AND CELLULAR BIOLOGY, June 2002, Vol. 22, No. 11 p. 3565-3576.

4 Markus Brielmeier, Jörg Schmidt Analyse der Funktion von Selenoproteinen Analysis of the functions of selenoproteins. Abteilung für Vergleichende Medizin. GSF-Forschungszentrum für Umwelt und Gesundheit, $\mathrm{GmbH}$ GSF 58-62.

http://www.gsf.de/neu/Wir ueber_uns/jahres bericht/2003/057_062_2003.pdf

5 Panel on Dietary Antioxidants and Related Compounds, Subcommittees on Upper Reference Levels of Nutrients and Interpretation and Uses of DRIs, Standing Committee on the Scientific Evaluation of Dietary Reference Intakes, Food and Nutrition Board Dietary Reference Intakes for Vitamin C, Vitamin E, Selenium, and Carotenoids Free Executive Summary, ISBN: 0-309-06935-1, (2000). http://www.nap.edu/catalog/9810.html

6 Thomson CD, Packer MA, Butler JA, Duffield AJ, O'Donaghue KL, Whanger PD Urinary selenium and iodine during pregnancy and lactation. J Trace Elem Med Biol (2001 Apr); 14(4):210-7.

7 Fox TE, Van den Heuvel EG, Atherton CA Dainty JR, Lewis DJ, Langford NJ, Crews HM, Luten JB, Lorentzen M, Sieling FW, van Aken-Schneyder P, Hoek M, Kotterman MJ, van Dael P, Fairweather-Tait SJ Bioavailability of selenium from fish, yeast and selenate: a comparative study in humans using stable isotopes. Eur J Clin Nutr (2004 Feb);58(2) 343-9.

8 Schrauzer GN: The nutritional significance, metabolism and toxicology of selenomethionine. Adv Food Nutr Res (2003);47:73-112.

9 Alfthan G, Xu GL, Tan WH, Aro A, Wu J, Yang YX, Liang WS, Xue WL, Kong LH: Selenium supplementation of children in a seleniumdeficient area in China: blood selenium levels and glutathione peroxidase activities. Biol Trace Elem Res. 2000 Feb;73(2):113-25.

10 Schrauzer GN. The nutritional significance, metabolism and toxicology of selenomethionine. Adv Food Nutr Res. 2003:47:73-112.

11 Kobayashi Y, Ogra Y, Ishiwata K, Takayama H, Aimi N, Suzuki KT Selenosugars are key and urinary metabolites for selenium excretion within the required to low-toxic range. Proc Natl Acad Sci USA (2002 Dec 10);99(25): 15932-6.

12 Huawei Zeng, Mary Briske-Anderson, Joseph P. Idso and Curtiss D. Hunt Nutrition and Disease. The Selenium Metabolite Methylselenol Inhibits the Migration and Invasion Potential of HT1080 Tumor Cells J. Nutr. 136 1528-1532, June 2006

13 Ishida T, Himeno K, Torigoe $Y$, Inoue M, Wak isaka O, Tabuki T, Ono H, Honda K, Mori T, Seike M, Yoshimatsu H, Sakata T: Selenium deficiency in a patient with Crohn's disease receiving long- term total parenteral nutrition. Intern Med (2003 Feb);42(2):154-7.

14 Sandstrom B, Davidsson L, Bosaeus I, Eriksson R, Alpsten $M$ : Selenium status and absorption of zinc (65Zn), selenium (75Se) and manganese $(54 \mathrm{Mn})$ in patients with short bowel syndrome. Eur J Clin Nutr (1990 Oct); 44(10):697-703.

15 Rannem T, Hylander E, Ladefoged K, Staun $M$, Tjellesen $L$, Jarnum S: The metabolism of [75Se]selenite in patients with short bowe syndrome. JPEN J Parenter Enteral Nutr (1996 Nov-Dec);20(6):412-6

16 Quilliot D, Walters E, Bohme P, Lacroix B Bonte JP, Fruchart JC, Drouin P, Duriez $P_{1}$ Ziegler O: Fatty acid abnormalities in chronic pancreatitis: effect of concomitant diabetes mellitus. Eur J Clin Nutr (2003 Mar):57(3): 496-503.

17 Chanoine JP: Selenium and thyroid function in infants, children and adolescents. Biofactors (2003);19(3-4):137-43.

18 Nezelof C, Bouvier R, Dijoud F: Multifocal myocardial necrosis: a distinctive cardiac lesion in cystic fibrosis, lipomatous pancreatic atrophy, and Keshan disease. Pediatr Patho Mol Med (2002 May-Jun);21(3):343-52

19 Burke MP, Opeskin K Fulminant heart failure due to selenium deficiency cardiomyopathy (Keshan disease). Med Sci Law (2002 Jan); 42(1):10-3.

20 Xu GL, Wang SC, Gu BO, Yang YX Song HB Xue WL, Liang WS, Zhang PY: Further investigation on the role of selenium deficiency in the aetiology and pathogenesis of Keshan disease. Biomed Environ Sci (1997 Sep);10 (2-3):316-26.

21 Fang W, Wu P, Hu R, Huang Z Environmental Se-Mo-B deficiency and its possible effects on crops and Keshan-Beck disease (KBD) in the Chousang area, Yao County, Shaanxi Province, China. Environ Geochem Health (2003 Jun);25(2):267-80.

22 Beck MA, Levander OA, Handy J: Selenium deficiency and viral infection. J Nutr (2003 May);133(5 Suppl 1):1463S-7S

23 Cermelli C, Vinceti M, Scaltriti E, Bazzani E, Beretti F, Vivoli G, Portolani M: Selenite inhibition of Coxsackie virus B5 replication: implications on the etiology of Keshan disease. $J$ Trace Elem Med Biol (2002); 16(1):41-6.

24 Moreno-Reyes R, Mathieu F, Boelaert M Begaux F, Suetens C, Rivera MT, Neve J, Perlmutter N, Vanderpas J: Selenium and iodine supplementation of rural Tibetan children affected by Kashin-Beck osteoarthropathy. Am J Clin Nutr (2003 Jul);78(1):137-44.

25 Eichholzer M, Gutzwiller F: Ernährungssituation in der Schweiz - Konsequenzen für die Vorbeugung. Schweiz Med Wochenschr 1997;127:1450-1456.

26 Sieber R., Haldimann M., Zimmerli B: Se-Status der Schweizerlnnen und Beitrag der Land- wirtschaft Agrarforschung 6(11), 441-444, 1999

27 Scientific American MEDICINE, (ISBN 0894554-009-2; IV DIET AND EXERCISE, Jan. 1998 (CD-ROM)

28 Berger MM, Shenkin A, Revelly JP, Roberts E, Cayeux MC, Baines M, Chiolero RL Copper, selenium, zinc, and thiamine balances during continuous venovenous hemodiafiltration in critically ill patients. Am J Clin Nutr (2004 Aug);80(2):410-6.

29 Heyland DK, Dhaliwal R, Suchner U, Berger MM: Antioxidant nutrients: a systematic review of trace elements and vitamins in the critically ill patient. Intensive Care Med (2005 Mar);31(3):327-37.

30 Andrew Tomkins. Assessing Micronutrient Status in the Presence of Inflammation 1. J. Nutr. 133:1649S-1655S, 2003.

31 Angstwurm MW, Engelmann L, Zimmermann T, Lehmann C, Spes CH, Abel P, Strauss R, Meier-Hellmann A, Insel R, Radké J, Schuttler J, Gartner R: Selenium in Intensive Care (SIC): results of a prospective randomized, placebo-controlled, multiple-center study in patients with severe systemic inflammatory response syndrome, sepsis, and septic shock. Crit Care Med (2007);35(1): 118-26.

32 Zimmermann T, Albrecht S, Hanke S, von Gagern G: Selen in der Intensivmedizin. Chirurgische Gastroenterologie 2000;16:54-57.

33 Gartner R, Gasnier BC. Selenium in the treatment of autoimmune thyroiditis. Biofactors (2003);19(3-4):165-70.

34 Duntas LH, Mantzou E, Koutras DA: Effects of a six month treatment with selenomethionine in patients with autoimmune thyroiditis. Eur J Endocrinol (2003 Apr);148(4):389-93.

35 Allam MF, Lucane RA: Selenium supplementation for asthma. Cochrane Database Syst Rev (2004)(2):CD003538.

36 European Respiratory Society. ERS response to the Commission Green Paper on healthy diets and physical activity. Issued 2006. ERS Headquarters Lausanne. 4 avenue Ste-Luce, $\mathrm{CH}-1003$ Lausanne, Switzerland, Website: www.ersnet.org

37 Shaheen SO, Newson RB, Rayman MP Wong AP, Tumilty MK, Phillips JM, Potts JF, Kelly FJ, White PT, Burney PG: Randomised double-blind, placebo-controlled trial of selenium supplementation in adult asthma. Thorax. 2007 Jan 18; [Epub ahead of print].

38 Heinle K, Adam A, Gradl M, Wiseman M, Adam O: [Selenium concentration in erythrocytes of patients with rheumatoid arthritis. Clinical and laboratory chemistry infection markers during administration of selenium] Selenkonzentration in den Erythrozyten bei Patienten mit rheumatoider Arthritis. Klinische und laborchemische Entzündungszeichen unter Supplementierung mit Selen. In: Med Klin (1997 Sep 15);92 Suppl 3:29-31.

39 Peretz A, Siderova V, Neve J: Selenium supplementation in rheumatoid arthritis investigated in a double blind, placebo-controlled trial. Scand J Rheumatol (2001);30(4):208-12.

40 Broome CS, McArdle F, Kyle JA, Andrews F, Lowe NM, Hart CA, Arthur JR, Jackson MJ: An increase in selenium intake improves immune function and poliovirus handling in adults with marginal selenium status. Am J Clin Nutr (2004 Jul);80(1):154-62.

41 Kasseroller R: Sodium selenite as prophylaxis against erysipelas in secondary lymphedema. Anticancer Res (1998 May-Jun);18(3C):222730.

42 Burbano X, Miguez-Burbano MJ, McCollister K, Zhang G, Rodriguez A, Ruiz P, Lecusay R, Shor-Posner G: Impact of a selenium chemo- 
prevention clinical trial on hospital admissions of HIV-infected participants. HIV Clin Trials (2002 Nov-Dec);3(6):483-91.

43 Shor-Posner G, Lecusay R, Miguez MJ, Moreno-Black G, Zhang G, Rodriguez N, Burbano X, Baum M, Wilkie F: Psychological burden in the era of HAART: impact of selenium therapy. Int J Psychiatry Med (2003);33(1): 55-69.

44 Hurwitz BE, Klaus JR, Llabre MM, et al. Suppression of human immunodeficiency virus type 1 viral load with selenium supplementation. A randomized controlled trial. Arch Intern Med 167:148-154, 2007.

45 Kupka R, Msamanga GI, Spiegelman D, Morris S, Mugusi F, Hunter DJ, Fawzi WW: Selenium status is associated with accelerated HIV disease progression among HIV-1-infected pregnant women in Tanzania. J Nutr (2004 Oct);134(10):2556-60

46 R Kupka, G I Msamanga, D Spiegelman3, N Rifai, D J Hunter and W W Fawzi: Selenium levels in relation to morbidity and mortality among children born to HIV-infected mothers. European Journal of Clinical Nutrition (2005);59, 1250-1258

47 Ärzte-Zeitung vom: 27.10.1992; Jahrgang 011; Nummer 193 (Internet).

48 Wollschlager S, Patzold K, Bulang T, Meissner $\mathrm{D}$, Porst $\mathrm{H}$ [Effect of preventive selenium administration on development of ERCPinduced acute pancreatitis] Med Klin (Munich) (1999 Oct 15) 94, Suppl 3:81-3.

49 Kuklinski B, Weissenbacher E, Fahnrich A: Coenzyme $\mathrm{Q} 10$ and antioxidants in acute myocardial infarction. Mol Aspects Med 1994; 15 Suppl:s143-7.

50 Kazuko Yoshizawa1, Alberto Ascherio1,2, J. Steven Morris3, Meir J. Stampfer1,2,4 Edward Giovannucci1,4, Connie K. Baskett3, Walter C. Willett1,2,4, and Eric B. Rimm Prospective Study of Selenium Levels in Toenails and Risk of Coronary Heart Disease in Men. Am J Epidemiol 2003;158:852-860.

51 Huang HY, Caballero B, Chang S, Alberg A, Semba R, Schneyer C. Wilson RF, Cheng TY Prokopowicz G, Barnes II GJ, Vassy J, Bass EB: Multivitamin/Mineral Supplements and Prevention of Chronic Disease. Evidence Report/Technology Assessment No. 139. (Prepared by The Johns Hopkins University Evidence-based Practice Center under Contract No. 290-02-0018). AHRQ Publication No. 06-E012. Rockville, MD: Agency for Healthcare Research and Quality. May 2006.

52 Cynthia D. Morris, Susan Carson. Routine Vitamin Supplementation to Prevent Cardiovascular Disease: A Summary of the Evidence for the U.S. Preventive Services Task Force. Ann Intern Med. 2003;139,56-70..

53 Akbaraly NT, Arnaud J, Hininger-Favier I, et al. Selenium and Mortality in the Elderly: Results from the EVA Study. Clin Chem 2005

54 Duffield-Lillico AJ, Slate EH, Reid ME, Turnbull BW, Wilkins PA, Combs GF, Park HK Gross EG, Graham GF, Stratton MS, Marshall JR, Clark LC: Selenium supplementation and secondary prevention of nonmelanoma skin cancer in a randomized trial. J Natl Cancer Inst (2003 Oct 1);95(19):1477-81.

55 Gerald F. Combs. Current Evidence and Research Needs to Support a Health Claim for Selenium and Cancer Prevention1 J. Nutr. 135:343-347, 2005

56 Yajun Hu, Richard V. Benya, Robert E. Carroll, and Alan M. Diamond: Allelic Loss of the Gene for the GPX1 Selenium-Containing Protein Is a Common Event in Cancer. J. Nutr. 2005;135:3021S-3024S.

57 Hanjing Zhuo, 1 Allan $H$. Smith, 1 and Craig Steinmaus1,2: Selenium and Lung Cancer: A
Quantitative Analysis of Heterogeneity in the Current Epidemiological Literature. Cancer Epidemiol Biomarkers Prev 2004;13(5). May 2004

58 Scott M. Lippman, Phyllis J. Goodman, Eric A. Klein, Howard L. Parnes, Ian M. Thomp son, Jr., et al. Designing the Selenium and Vitamin E Cancer Prevention Trial (SELECT) Journal of the National Cancer Institute, Vol. 97, No. 2, January 19, 2005, 94-102.

59 Haojie Li, Meir J. Stampfer, Edward L. Giovannucci, J. Steven Morris, Walter C. Willett, J. Michael Gaziano, Jing Ma: A Prospective Study of Plasma Selenium Levels and Prostate Cancer Risk. Journal of the Nationa Cancer Institute, Vol. 96, No. 9, May 5, 2004 696-703.

60 Piet A. van den Brandt, Maurice P. A Zeegers, Peter Bode, and R. Alexandra Goldbohm. Toenail Selenium Levels and the Subsequent Risk of Prostate Cancer: A Prospective Cohort Study. Cancer Epidemiology, Biomarkers \& Prevention September 2003:Vol. 12:866-871.

61 William G. Nelson, Angelo M. De Marzo, and William B. Isaacs. Mechanisms of disease. Prostate Cancer. N Engl J Med 2003;349 366-81.

62 William G. Nelson, Angelo M. De Marzo, and William B. Isaacs. Mechanisms of disease. Prostate Cancer. N Engl J Med 2003;349: 366-81.

63 Bjelakovic G, Nikolova D, Simonetti RG, Gluud C: Antioxidant supplements for prevention of gastrointestinal cancers: a systematic review and meta-analysis. Lancet 2004 Oct 2-8;364(9441):1219-28.

64 Yajun Hu, Richard V. Benya, Robert E. Carroll, and Alan M. Diamond: Allelic Loss of the Gene for the GPX1 Selenium-Containing Protein Is a Common Event in Cancer. J. Nutr. 2005;135:3021S-3024S.

65 Yu SY, Zhu YJ, Li WG: Protective role of selenium against hepatitis $B$ virus and primary liver cancer in Qidong. Biol Trace Elem Res (1997 Jan);56(1):117-24.

66 Mark SD, Oiao YL, Dawsey SM, Wu YP, Katk H, Gunter EW, Fraumeni JF Blot WJ, Dong ZW, Taylor PR: Prospective study of serum selenium levels and incidentesophageal and gastric cancers. J Natl Cancer Inst (2000 Nov 1):92(21):1753-63.

67 NATIONAL INSTITUTES OF HEALTH STATEOF-THE-SCIENCE CONFERENCE STATEMENT Multivitamin/Mineral Supplements and Chronic Disease Prevention May 15-17 2006.

68 Salonen JT Salonen R Seppanen K Rinta-Kiik ka S Kuukka M Korpela H Alfthan G Kantola M Schalch W: Effects of antioxidant supplementation on platelet function: a randomized pair-matched, placebo-controlled doubleblind trial in men with low antioxidant status [see comments]. In: Am J Clin Nutr (1991 May);53(5):1222-1229

69 Nyyssonen K Porkkala E Salonen R Korpela H Salonen JT: Increase in oxidation resistance of atherogenic serum lipoproteins following antioxidant supplementation:a randomized double-blind placebo-controlled clinical trial. In: Eur J Clin Nutr (1994 Sep);48(9):633-642.

70 Brude IR Drevon CA Hjermann I Seljeflot Lund-Katz S Saarem K Sandstad B Solvoll K Halvorsen B Arnesen H Nenseter MS: Peroxidation of LDL from combined-hyperlipidemic male smokers supplied with omega-3 fatty acids and antioxidants. In: Arterioscler Thromb Vasc Biol (1997);17(11):2576- 88.

71 Galan P, Preziosi P, Monget AL, Richard MJ Arnaud J, Lesourd B, Girodon F, Alferez MJ Bourgeois C, Keller Favier A, Hercberg S: Effects of trace element and/or vitamin sup- plementation on vitamin and mineral status, free radical metabolism and immunological markers in elderly long term-hospitalized subjects. Geriatric Network MIN. VIT. AOX. In: Int J Vitam Nutr Res (1997);67(6):450-460.

72 Porter JM, Ivatury RR, Azimuddin K, Swami $\mathrm{R}$ : Antioxidant therapy in the prevention of organ dysfunction syndrome and infectious complications after trauma: early results of a prospective randomized study. Am Surg (1999 May);65(5):478-83.

73 Berger MM, Eggimann P, Heyland DK, Chiolero RL, Revelly JP, 'Day A, Raffoul W' Shenkin A: Reduction of nosocomial pneumonia after major burns by trace element supplementation: aggregation of two randomised trials. Crit Care (2006);10(6):R153.

74 Berger MM, Reymond MJ, Shenkin A, Rey F, Wardle C, Cayeux C, Schindler C, Chiolero $\mathrm{RL}$ : Influence of selenium supplements on the post-traumatic alterations of the thyroid axis: a placebo-controlled trial. Intensive Care Med (2001 Jan);27(1):91-100.

75 Girodon F. Lombard M. Galan P. BrunetLecomte P. Monget AL. Arnaud J. Preziosi P. Hercberg S: Effect of micronutrient supplementation on infection in institutionalized elderly subjects: a controlled trial. In: Ann Nutr Metab (1997);41(2):98-107.

76 Girodon F. Galan P. Monget AL. BoutronRuault MC. Brunet-Lecomte P. Preziosi P. Arnaud J. Manuguerra JC. Herchberg S: Impact of trace elements and vitamin supplementation on immunity and infections in institutionalized elderly patients: a randomized controlled trial. MIN. VIT. AOX geriatric network In: Arch Intern Med (1999 Apr 12): 159(7):748-754.

77 Johnson MA, Porter KH: Micronutrient supplementation and infection in institutionalized elders. In: Nutr Rev (1997 Nov);55(11 Pt 1): 400-4.

78 Weijl NI, Elsendoorn TJ, Lentjes EG, Hopman GD, Wipkink-Bakker A, Zwinderman AH, Cleton FJ, Osanto S: Supplementation with antioxidant micronutrients and chemotherapy-induced toxicity in cancer patients treated with cisplatin-based chemotherapy: a randomised, double-blind, placebo-controlled study. Eur J Cancer (2004 Jul);40(11):171323.

79 Marwan G. Fakih1, Lakshmi Pendyala1, Patrick F. Smith1, Patrick J. Creaven1, Mary E. Reid, Vladimir Badmaev, Rami G. Azrak, Joshua D. Prey1, David Lawrence and Youcef M: Rustum Cancer Therapy: Clinical. A Phase I and Pharmacokinetic Study of Fixed-Dose Selenomethionine and Irinotecan in Solid Tumors. Clinical Cancer Research Vol. 12, 1237-1244, February 2006.

80 Dennert G, Horneber M: Selenium for alleviating the side effects of chemotherapy, radiotherapy and surgery in cancer patients. Cochrane Database of Systematic Reviews 2006, Issue 3. Art. No.: CD005037. DOI: 10.1002/14651858.CD005037. pub2

81 Jacobs ET, Jiang R, Alberts DS, Greenberg ER, Gunter EW, Karagas MR, Lanza E, Ratnasinghe $L$, Reid ME, Schatzkin A, SmithWarner SA, Wallace K, Martinez ME:Selenium and colorectal adenoma: results of a pooled analysis. J Natl Cancer Inst (2004 Nov 17);96(22):1669-75.

\section{Korrespondenzadresse:}

Prof. Dr. med. Reinhard Saller

UniversitätsSpital Zürich

Dep. für Innere Medizin

Institut für Naturheilkunde

Rämistrasse 100, CH-8091 Zürich

reinhard.saller@usz.ch 